\title{
Measuring instrument constructs for knowledge management system governance using Rasch measurement model
}

\begin{abstract}
Knowledge management system (KMS) has become an important asset to the organisation. KMS acting as a tool in supporting organisation knowledge management (KM) practices. The advancement of internet technology makes it easier for organisation KMS working in a collaborative manner. With the rapid development of KMS in organisation, collaboration among these KMS would make a smooth sharing of information. This research aims to scrutinize the governance mechanism of KMS used in managing and controlling the agency KMS. From the previous study, the governance mechanism had been identified based on the development of the conceptual framework. This study investigates the relationships of governance mechanism that consist of two main sub-systems: (1) KMS governance; and (2) collaborative KMS. A survey was conducted by distributing a questionnaire to 25 respondents from eight (8) Malaysian government agencies. The respondent comprises of Community of Practice (CoP), Subject Matter Expert, ICT experts and KM User. The aim of this paper is to determine the validity and reliability of each dimension in the questionnaire using the Rasch Measurement Model (RMM) analysis. The survey was developed comprises 2 sections consisting of 40 items. A total of 21 responses were received. The RMM is used to measure the quality control of item dimensions in the instrument. As a result, almost all the items in the questionnaire constructs were statistically reliable and valid for further analysis, and a few items need to be revised and changed. This specifies that the respondents are reliable and the items are reasonable for this study.
\end{abstract}

Keyword: Collaborative; Governance; Knowledge management system 